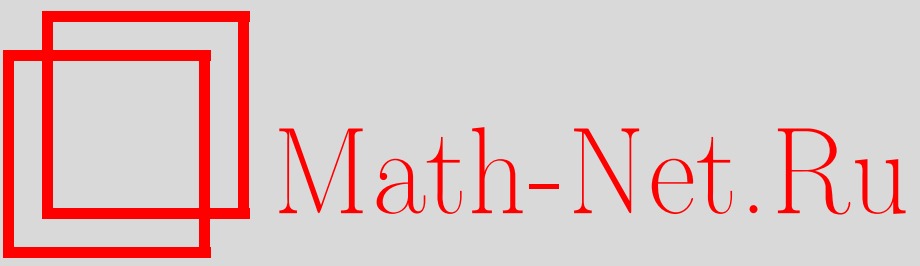

В. П. Барашев, В. В. Белов, А. С. Вшивцев, А. Г. Кисунько, Некоторые особенности термодинамики идеальных систем с нелинейным взаимодействием, $Т M \Phi$, 1998, том 116, номер 3, 431-441

DOI: https://doi.org/10.4213/tmf914

Использование Общероссийского математического портала Math-Net.Ru подразумевает, что вы прочитали и согласны с пользовательским соглашением

http: //www . mathnet.ru/rus/agreement

Параметры загрузки:

IP : 3.85 .5 .30

26 апреля 2023 г., 16:40:42 


\author{
ТЕОРЕТИЧЕСКАЯ \\ И МАТЕМАТИЧЕСКАЯ \\ ФИЗИКА \\ Том 116, № 3 \\ сентябрь, 1998
}

(C) 1998 г. В.П. Барашев ${ }^{*}$ В.В. Белов ${ }^{\dagger}$,

А.С. Вшивцев*, А.Г. Кисунько*

\title{
НЕКОТОРЫЕ ОСОБЕННОСТИ ТЕРМОДИНАМИКИ ИДЕАЛЬНЫХ СИСТЕМ С НЕЛИНЕЙНЫМ ВЗАИМОДЕЙСТВИЕМ
}

Проведено сравнительное изучение термодинамических свойств для двух двумерных систем. Одна из них отвечает связанному состоянию двумерного гармонического осциллятора, а вторая - нелинейному ангармоническому осциллятору. Приведен спектр для гармонического осциллятора, а для нелинейного осциллятора впервые приведены результаты расчетов ВКБ-спектра и волновых функций. Показано, что $\Omega$-потенциалы имеют существенно различное поведение в области высоких температур. Это может быть замечено, например, при исследовании температурной зависимости теплоемкости в планарных анизотропных средах.

Изучение термодинамических свойств различных систем позволяет в значительной мере расширить наши представления о природе того или иного состояния вешества.

Именно исследование термодинамических свойств электронного газа в магнитном поле привело к предсказанию эффекта осцилляций намагниченности в металлах [1-3]. До настояшего времени эта тема не исчерпала себя и продолжает интересовать исследователей [4-6]. Одна из причин, по которой сохраняется интерес к этой проблеме, - исследование явления высокотемпературной сверхпроводимости, а также в связи с этим детальное рассмотрение эффектов, происходящих в планарных структурах. Здесь имеется широкий круг проблем, впервые сформулированных в работах Лифшица [7]. Одной из таких проблем является учет нелинейных эффектов взаимодействия электронного газа с кристаллической решеткой, а также анализ физических следствий, к которым приводит нелинейность взаимодействия.

Традиционный подход к изучению взаимодействия, как правило, основывается на построении аппроксимирующего потенциала взаимодействия. Далее для этого потенциала решается спектральная задача. В нерелятивистском случае находится спектр уравнения Шредингера в потенциалах, моделирующих физические особенности поведения

\footnotetext{
* Московский государственный институт радиотехники, электроники и автоматики (технический университет), Москва, Россия

${ }^{\dagger}$ Московский государственный институт электроники и математики (технический университет), Москва, Россия
} 
электронов в поле кристаллической решетки твердого тела. При рассмотрении уравнения Шредингера, как правило, ограничиваются гармоническим приближением, что обусловлено математическими трудностями нахождения точного спектра многомерной задачи с учетом ангармонизма потенциалов. К сожалению, обоснование использования гармонического приближения не всегда бывает корректным и неясно, насколько в модифицированной таким образом задаче сохраняются физические особенности исходной задачи.

С учетом сказанного мы проанализируем поведение термодинамического потенциала, основываясь на рассмотрении двух планарных задач. Гамильтониан первой задачи

$$
\widehat{H}_{1}=\frac{\widehat{p}_{x}^{2}}{2 m}+\frac{\widehat{p}_{y}^{2}}{2 M}+U_{1}(x, y)
$$

где

$$
U_{1}(x, y)=\frac{k}{2}\left(x^{2}+y^{2}\right)+\varepsilon x y, \quad \varepsilon>0
$$

Для второй задачи

$$
\widehat{H}_{2}=\frac{\widehat{p}_{x}^{2}}{2 m}+\frac{\widehat{p}_{y}^{2}}{2 m}+U_{2}(x, y)
$$

где

$$
U_{2}(x, y)=\frac{U_{0}}{4}\left(x^{4}+y^{4}\right)+\frac{\varepsilon U_{0}}{2} x^{2} y^{2}, \quad \varepsilon>0
$$

$U_{0}$ - параметр, учитьвающий размерную зависимость потенциальной энергии.

Гамильтониан первого типа известен из курса квантовой механики [8], а также обсуждается в обычной классической механике как пример двух взаимодействуюших осцилляторов. Характерной особенностью этой задачи являются возможность разделения переменных в уравнении Шредингера и ее точное решение. Соответствующий спектр имеет вид

$$
E_{n, \nu}^{(1)}=E_{01}\left(a_{1}\left(n+\frac{1}{2}\right)+b_{1}\left(\nu+\frac{1}{2}\right)\right) .
$$

Здесь $E_{01}=\hbar \omega / \sqrt{2}, \omega=\sqrt{k / m}, n, \nu=0,1,2 \ldots$, а константы $a_{1}$ и $b_{1}$ выражаются следуюшим образом через массы $m$ и $M$ осцилляторов и параметр $\varepsilon$ :

$$
\begin{aligned}
& a_{1}=\left[1+\frac{m}{M}+\sqrt{\left(1-\frac{m}{M}\right)^{2}+\frac{4 m \varepsilon^{2}}{M k^{2}}}\right]^{1 / 2}, \\
& b_{1}=\left[1+\frac{m}{M}-\sqrt{\left(1-\frac{m}{M}\right)^{2}+\frac{4 m \varepsilon^{2}}{M k^{2}}}\right]^{1 / 2} .
\end{aligned}
$$

Заметим, что для приложения к задаче об осцилляциях намагниченности гамильтониан (1) фигурирует достаточно часто [6]. Более того, обсуждается возможность приближения таким гамильтонианом широкого класса гамильтонианов с нелинейным типом взаимодействия [9]. Для того чтобы решить вопрос о правомерности такого подхода, 
мы сравним термодинамические потенциалы электронного газа с гамильтонианами (1) и (2) и установим области параметров, в которых возможно приближение одной модели другой.

При $\mu<0$ ( $\mu$ - химический потенциал) $\Omega$-потенциал идеального электронного газа со спектром (3) вычисляется просто и имеет вид

$$
\Omega_{1-}=\frac{4}{\beta} \sum_{n=1}^{\infty} \frac{(-1)^{n}}{n} \frac{\exp (\mu \beta n)}{\operatorname{sh}\left(\beta E_{01} a_{1} n / 2\right) \operatorname{sh}\left(\beta E_{01} b_{1} n / 2\right)}
$$

При $\mu>0$ этот же $\Omega$-потенциал задается соотношением

$$
\begin{aligned}
\Omega_{1+}= & \frac{4}{\beta}\left\{\sum_{n=1}^{\infty} \frac{(-1)^{n}}{n} \frac{\exp (-\mu \beta n)}{\operatorname{sh}\left(\beta E_{01} a_{1} n / 2\right) \operatorname{sh}\left(\beta E_{01} b_{1} n / 2\right)}+\right. \\
& +\frac{2 \mu\left[\left(\beta E_{01} / 2\right)^{2}\left(a_{1}^{2}+b_{1}^{2}\right)-(\mu \beta)^{2}-\pi^{2}\right]}{3 E_{01}^{2} a_{1} b_{1} \beta}+ \\
& +\sum_{n=1}^{\infty} \frac{2(-1)^{n}}{n}\left[\sin \left(\frac{2 \pi n \mu}{E_{01} a_{1}}\right) \operatorname{sh}^{-1}\left(\frac{2 \pi^{2} n}{E_{01} b_{1} \beta}\right) \sin ^{-1}\left(\frac{\pi n a_{1}}{b_{1}}\right)+\right. \\
& \left.\left.+\sin \left(\frac{2 \pi n \mu}{E_{01} b_{1}}\right) \operatorname{sh}^{-1}\left(\frac{2 \pi^{2} n}{E_{01} a_{1} \beta}\right) \sin ^{-1}\left(\frac{\pi n b_{1}}{a_{1}}\right)\right]\right\} .
\end{aligned}
$$

Выражение (5) хорошо известно, и, в частности, исследователями обсуждаются проблемы суммирования быстроосциллирующих рядов, стоящих в (5). Мы обратим внимание лиш на тот факт, что в высокотемпературном пределе $(\beta \rightarrow 0)$ из $(4)$ и $(5)$ следует асимптотическое поведение для $\Omega$-потенциала идеального газа:

$$
\left.\Omega_{1 \pm}\right|_{\beta \rightarrow 0}=-\frac{12 \zeta(3)}{E_{01}^{2} a_{1} b_{1} \beta^{3}} \pm \frac{4 \pi^{2} \mu}{3 E_{01}^{2} a_{1} b_{1} \beta^{2}}+O\left(\beta^{-1}\right) .
$$

Заметим, что осциллируюшая часть $\Omega$-потенциала в (5) фактически является двоякопериодической функцией с периодами, связанными с параметрами $a_{1}$ и $b_{1}$. Арифметическая природа отношения $a_{1} / b_{1}$ обусловливает расходимость ряда в (5). Здесь речь фактически идет о свойствах осциллируюшей части функции $\Omega$ на двумерной по $a_{1}$ и $b_{1}$ решетке, изоморфной тору. Становится ясно, что устранить эту расходимость принципиально невозможно, т.к. это одно из характерных свойств рассматриваемой системы. В данном случае такое обстоятельство аналогично явлению резонансов инвариантных торов в интегрируемой системе $[10,11]$.

Выбор модельного гамильтониана (2) обусловлен двумя обстоятельствами. Во-первых, такие потенциалы взаимодействия с сильным ангармонизмом описывают свойства электронов в квазинульмерных системах (квантовые ямы и квантовые точки) [4, 6]. Во-вторых, система с потенциалом (2) является простейшим примером классической и, возможно, квантовой неинтегрируемости. Классическая неинтегрируемость (отсутствие дополнительного первого интеграла) при $\varepsilon \neq 0,1,3$ доказана в работе [12]. 
Численному исследованию структуры фазового пространства классической системы с гамильтонианом $H_{2}(p, q)$, где $p=\left(p_{x}, p_{y}\right), q=(x, y)$, а уравнения движения имеют вид

$$
\dot{p}=-H_{q}, \quad \dot{q}=H_{p},
$$

посвящено значительное число работ [13].

Было установлено, что в широком диапазоне параметров, энергии $E>0$ и константы связи $\varepsilon \geqslant 0$ система (7) относится к классу систем с "мягким хаосом", т.е. содержит как регулярную, так и стохастическую компоненты поведения фазовых траекторий, включая большие значения параметра $\varepsilon$ [14].

Соответствуюшая квантовая спектральная задача

$$
\widehat{H}_{2}(\varepsilon) \psi_{E}=E \psi_{E}, \quad \psi_{E} \in L_{2}\left(\mathbf{R}_{x, y}^{2}\right)
$$

имеет чисто дискретный спектр при любом положительном значении параметра $\varepsilon$ (для $\varepsilon=\infty$ с потенциалом $V(x, y, \infty)=x^{2} y^{2} / 4$ этот факт установлен в работе [15]).

Неинтегрируемость системы (7) не позволяет получить какую-либо информацию о поведении спектра и волновых функций задачи (8) в рамках стандартного квазиклассического приближения $(\hbar \rightarrow 0)$ - многомерного метода Венцеля-Крамерса-Бриллюэна (ВКБ)-Маслова.

Тем не менее квазиклассический подход к спектральной квантовой задаче (8), основанный на теории комплексного ростка Маслова (комплексный метод ВКБ, развитый в работах [16-18]), позволяет выделить в спектре гамильтониана $H_{2}(2)$ ту его часть, которая порождается регулярным движением вдоль устойчивых фазовых орбит соответствуюшей классической системы (7).

Здесь мы приведем, следуя статье [18], результаты расчета квазиклассических уровней энергии и соответствующих им волновых функций - спектральных серий (квазимод), ассоциированных со специальными семействами устойчивых фазовых орбит $\Lambda_{j}^{1}(E), j=1,2,3,4$, на уровне энергии $E$ классической функции Гамильтона. Эти орбиты порождены глобальной дискретной симметрией потенциала $V(x, y, \varepsilon)$ относительно замен: 1) $x \rightarrow x, y \rightarrow-y, 2) x \rightarrow-x, y \rightarrow y, 3) x \rightarrow-y, y \rightarrow x, 4) x \rightarrow y, y \rightarrow-x$, соответственно, и имеют вид

$$
\begin{aligned}
& \Lambda_{1}^{1}(E)=\left\{y=0, \quad p_{y}=0, \quad \frac{x^{4}}{4}+\frac{p_{x}^{2}}{2}=E\right\}, \\
& \Lambda_{2}^{1}(E)=\left\{x=0, \quad p_{x}=0, \quad \frac{y^{4}}{4}+\frac{p_{y}^{2}}{2}=E\right\}, \\
& \Lambda_{3}^{1}(E)=\left\{y=x, \quad p_{y}=p_{x}, \quad \frac{x^{4}(1+\varepsilon)}{2}+p_{x}^{2}=E\right\}, \\
& \Lambda_{4}^{1}(E)=\left\{y=-x, \quad p_{y}=-p_{x}, \quad \frac{y^{4}(1+\varepsilon)}{2}+p_{y}^{2}=E\right\} .
\end{aligned}
$$


Периодические движения вдоль этих фазовых орбит - прямолинейные либрации очевидно, описываются эллиптическими функциями. Например, для $\Lambda_{1}^{1}(E)$ имеем

$$
x(t)=-\alpha \operatorname{cn}\left(\frac{2 \tau \mathbf{K}(1 / \sqrt{2})}{\pi}, \frac{1}{\sqrt{2}}\right), \quad p=\dot{x}(t), \quad \tau=\frac{2 \pi}{T(E)} t,
$$

где $T(E)=4 \mathbf{K}(1 / \sqrt{2}) / \alpha-$ период движения, $\alpha=(4 E)^{1 / 4}, \mathbf{K}(1 / \sqrt{2})-$ полньй эллиптический интеграл.

Квазиклассические уровни энергии, отвечаюшие фазовым кривым $\Lambda_{1,2}^{1}(E)$, имеют вид

$$
\begin{aligned}
E_{n, \nu}^{(1,2)}(\hbar, \varepsilon)= & U_{0}^{1 / 3}\left(\frac{3 \pi \hbar}{2^{5 / 2} \mathbf{K}(1 / \sqrt{2})}\right)^{4 / 3}\left[n+\frac{1}{2}+(m+\theta(\varepsilon))\left(\nu+\frac{1}{2}\right)\right]^{4 / 3}+ \\
& +O\left(\hbar^{3 / 2}\right), \quad \hbar \rightarrow 0 .
\end{aligned}
$$

Здесь $n, \nu=0,1,2, \ldots$ - квантовые числа, а целочисленный индекс $m=0,1,2, \ldots$ - номер зоны устойчивости по параметру $\varepsilon \in \Omega_{m}=(m(m+1),(m+1)(2 m+1))$ уравнения Хилла, к которому сводится система уравнений в нормальных вариациях в окрестности движения вдоль фазовых орбит $\Lambda_{1,2}^{1}(E)$ :

$$
\frac{d^{2} z}{d \tau^{2}}+\Omega(\tau) z=0, \quad \tau=\frac{2 \pi t}{T(E)} \quad(\bmod 2 \pi) .
$$

В уравнении (12) частота

$$
\Omega(\tau)=\varepsilon \frac{4 \mathbf{K}(1 / \sqrt{2})}{\pi^{2}} \mathrm{cn}^{2}\left(\frac{2 \mathbf{K}(1 / \sqrt{2})}{\pi} \tau, \frac{1}{\sqrt{2}}\right),
$$

а показатель Флоке

$$
\theta(\varepsilon)=\arccos \left(\sqrt{2} \cos \left(\frac{\pi}{4} \sqrt{1+8 \varepsilon}\right)\right), \quad \varepsilon \in \Omega_{m} .
$$

Кривым $\Lambda_{3,4}^{1}(E)$ отвечают серии квазиклассических уровней энергии, имеющие вид

$$
E_{n, \nu}^{(3,4)}(\hbar, \varepsilon)=(1+\varepsilon)^{1 / 3} E_{n, \nu}^{(1,2)}\left(\hbar, \frac{3-\varepsilon}{1+\varepsilon}\right), \quad \hbar \rightarrow 0 .
$$

Для этих кривых условие орбитальной устойчивости в линейном приближении сводится к неравенствам $1<\varepsilon<3$.

Приведем явные формулы для квазиклассических волновых функций вне окрестностей проекций фокальных точек $(x= \pm \alpha, y=0)$, когда $|x|<\alpha, y=O(\sqrt{\hbar})$ :

$$
\begin{aligned}
\psi_{n, \nu}(x, y, \varepsilon, \hbar)= & \frac{1}{\sqrt{T(E)} \sqrt{p(x, E)}} \times \\
& \times \cos \left(\frac{1}{\hbar} \int_{-\alpha}^{x} p\left(x_{1}, E\right) d x_{1}-\frac{\pi}{4}-\left(\nu+\frac{1}{2}\right) \frac{\beta_{f}(\varepsilon)}{2}\right) \times \\
& \times\left.\Phi_{\nu}\left(\tau(x), \frac{y}{\sqrt{\hbar}}\right) \exp \left[i\left(\nu+\frac{1}{2}\right) \frac{\beta_{f}}{2}\right]\right|_{E=E_{n, \nu(\hbar, \varepsilon)}},
\end{aligned}
$$


где

$$
\begin{aligned}
\Phi_{\nu}\left(\tau(x), \frac{y}{\sqrt{\hbar}}\right)= & N_{\nu}(\hbar)[z(\tau(x))]^{-1 / 2} \exp \left[i \frac{y^{2} \dot{z}(\tau(x))}{2 \hbar z(\tau(x))}-i \nu \operatorname{Arg} z(\tau(x))\right] \times \\
& \times H_{\nu}\left(\frac{y}{\sqrt{\hbar}|z(\tau(x))|}\right)
\end{aligned}
$$

для любого $\varepsilon$, принадлежашего $m$-зоне устойчивости уравнения $(12)$. Здесь $p(x, E)=$ $\sqrt{2\left(E-x^{4} / 4\right)}, z=z(\tau)$ - устойчивое фолоке-решение уравнения $(12), \tau(x)$ - гладкое решение (10) относительно $x, \quad|x|<\alpha$ при $\tau \neq 0(\bmod \pi), \quad \dot{z}(\tau)=d z / d \tau, \quad N_{\nu}(\hbar)=$ $\left[(\pi \hbar)^{1 / 4} 2^{\nu / 2} \sqrt{\nu !}\right]^{-1}, \beta_{f}(\varepsilon)=m+\theta(\varepsilon), H_{\nu}(x)-$ полиномы Эрмита.

Функции (14) локализованы при $\hbar \rightarrow 0$ в малой окрестности оси $x,|x| \leqslant \alpha$, осциллируя вдоль $x$ с частотой порядка $1 / \hbar$ аналогично одномерным ВКБ-функциям, и экспоненциально убывают по $y$ (в силу свойства $\operatorname{Im}(\dot{z} / z)>0$ ), осциллируя с частотой порядка $\sqrt{1 / \hbar}, \hbar \rightarrow 0$, определяемой нулями многочленов Эрмита в $(15)$.

Результатом нашего рассмотрения задачи (8) являются следуюшие утверждения.

1. Квазиклассические спектральные серии уравнения Шредингера с гамильтонианом (2), порож денные периодическими орбитами (9), имеют следуюшую обшую структуру (с точностью до $\left.\hbar^{3 / 2}\right)$ :

$$
E_{n, \nu}^{(1,2)}(\hbar, \varepsilon)=E_{0}\left[n+\frac{1}{2}+\beta_{f}(\varepsilon)\left(\nu+\frac{1}{2}\right)\right]^{4 / 3}
$$

где

$$
\begin{gathered}
E_{0}=U_{0}^{1 / 3}\left(\frac{3 \pi \hbar}{2^{5 / 2} \mathbf{K}(1 / \sqrt{2})}\right)^{4 / 3}, \\
\beta_{f}(\varepsilon)=m+\theta(\varepsilon), \quad \varepsilon \in \Omega_{m}, \quad n, \nu=0,1,2, \ldots .
\end{gathered}
$$

2. Процедура квазиклассического квантования комплексным методом ВКБ такова, что используются только устойчивые замкнутые фазовые орбиты, т.е. стохастическая компонента классического движения не рассматривается.

3. С помощью процедуры ВКБ-квантования найдены волновые функции (14).

Далее мы используем серию собственных значений для либрации вдоль направления $x=0$. Вычисление $\Omega$-потенциала для других серий выполняется аналогично.

Рассмотрим возможность установления соответствия между результатами, полученными на основе гамильтонианов (2) и (1). Явные выражения для спектральных серий (3) и (16) показывают, что единственным связуюшим фактором является определение спектров (1) и (2) на двумерных решетках. Степенная зависимость по $n$ и $\nu$ в (3) и (16), как и следовало ожидать, существенно различная. Если для двумерного гармонического осциллятора мы имеем линейную функцию по этим параметрам, то для гамильтониана (2) - степенную функцию с показателем 4/3 (в одномерном аналоге модели $(2)$, когда $U(x)=x^{4}$, возникает такая же степень $\left.[17,19]\right)$. 
Исследуем $\Omega$-потенциал электронного газа со спектром типа (16). Известно, что $\Omega$-потенциал представим в виде [20]

$$
\Omega=-\frac{1}{\beta} \sum_{n, \nu=0}^{\infty} \ln \left[1+e^{\left(\mu-E_{n, \nu}\right) \beta}\right] .
$$

Используем интегральное представление [21]

$$
\ln (1+\alpha x)=\frac{1}{2 \pi} \int_{c-i \infty}^{c+i \infty} \frac{\pi}{s \sin \pi s}(\alpha x)^{s} d s,
$$

а также формулу [21]

$$
\exp \left(-\alpha x^{h_{1}}\right)=\frac{1}{2 \pi} \int_{c_{1}-i \infty}^{c_{1}+i \infty} h_{1}^{-1} \alpha^{-s_{1} / h_{1}} \Gamma\left(\frac{s_{1}}{h_{1}}\right) x^{-s_{1}} d s_{1} .
$$

В результате мы найдем следуюшее интегральное представление для $\Omega$-потенциала:

$$
\begin{aligned}
\Omega= & -\frac{1}{\beta 2 \pi i} \int_{c-i \infty}^{c+i \infty} d s \frac{\pi e^{\mu \beta s}}{s \sin \pi s} \frac{3}{8 \pi i} \int_{c_{1}-i \infty}^{c_{1}+i \infty} d s_{1}\left(E_{0} \beta s\right)^{-3 s_{1} / 4} \Gamma\left(\frac{3 s_{1}}{4}\right) \times \\
& \times \zeta\left(s_{1}, \beta_{f}(\varepsilon), \frac{\beta_{f}(\varepsilon)+1}{2}\right),
\end{aligned}
$$

где

$$
\zeta(s, a, b)=\sum_{n, k=0}^{\infty}(n+a k+b)^{-s}
$$

(дополнительные свойства этой функции изложены в приложении).

Для выгисления контурных интегралов необходимо выделить особые точки функции (21). Это можно сделать, воспользовавшись формулой суммирования [22]

$$
\sum_{n=0}^{\infty} f(n)=\frac{1}{2} f(0)+\int_{0}^{\infty} f(\tau) d \tau+i \int_{0}^{\infty} \frac{f(i t)-f(-i t)}{e^{2 \pi t}-1} d t
$$

При этом получается следуюшее представление для функции (21):

$$
\begin{aligned}
\zeta(s, a, b)= & \frac{1}{4 b^{s}}+\frac{a+1}{2(s-1) a b^{s-1}}+\frac{1}{a(s-1)(s-2) b^{s-2}}+ \\
& +\int_{0}^{\infty} \frac{\sin [s \operatorname{arctg}(t / b)]}{\left(e^{2 \pi t}-1\right)\left(t^{2}+b^{2}\right)^{s / 2}} d t+\int_{0}^{\infty} \frac{\sin [s \operatorname{arctg}(a t / b)]}{\left(e^{2 \pi t}-1\right)\left((a t)^{2}+b^{2}\right)^{s / 2}} d t+ \\
& +\frac{2}{a(s-1)} \int_{0}^{\infty} \frac{\sin [(s-1) \operatorname{arctg}(t / b)]}{\left(e^{2 \pi t}-1\right)\left(t^{2}+b^{2}\right)^{(s-1) / 2}} d t+ \\
& +\frac{2}{s-1} \int_{0}^{\infty} \frac{\sin [(s-1) \operatorname{arctg}(a t / b)]}{\left(e^{2 \pi t}-1\right)\left((a t)^{2}+b^{2}\right)^{(s-1) / 2}} d t+ \\
& +2 \int_{0}^{\infty} \frac{d \tau}{\left(e^{2 \pi \tau}-1\right)} \int_{0}^{\infty}\left\{\frac{\cos [s \operatorname{arctg}((a \tau-t) / b)]}{\left[(a \tau-t)^{2}+b^{2}\right]^{s / 2}}-\right. \\
& \left.-\frac{\cos [s \operatorname{arctg}((a \tau+t) / b)]}{\left[(a \tau+t)^{2}+b^{2}\right]^{s / 2}}\right\} \frac{d t}{e^{2 \pi t}-1}
\end{aligned}
$$


Из равенства (23) видно, что функция $\zeta(s, a, b)$ имеет простые полюсы в точках $s=1$ и $s=2$ с вычетами

$$
\operatorname{Res}_{s=1} \zeta(s, a, b)=\frac{a+1-2 b}{2 a}, \quad \underset{s=2}{\operatorname{Res}} \zeta(s, a, b)=\frac{1}{a} .
$$

Учитывая особенности Г-функции, находим

$$
\begin{aligned}
\Omega= & -\frac{1}{\beta 2 \pi i} \int_{c-i \infty}^{c+i \infty} d s \frac{\pi e^{\mu \beta s}}{s \sin (\pi s)} \frac{3}{4}\left[\frac{\sqrt{\pi}}{2 \beta_{f}(\varepsilon)}\left(E_{0} \beta s\right)^{-3 / 2}+\right. \\
& \left.+\sum_{n=0}^{\infty} \frac{(-1)^{n}}{n !}\left(E_{0} \beta s\right)^{n} \zeta\left(-\frac{4 n}{3}, \beta_{f}(\varepsilon), \frac{\beta_{f}(\varepsilon)+1}{2}\right)\right] .
\end{aligned}
$$

Для вычисления интегралов вида

$$
I(r)=-\frac{1}{2 \pi i} \int_{c-i \infty}^{c+i \infty} d s \frac{\pi e^{\mu \beta s} s^{r}}{\sin (\pi s)}
$$

заметим, что

$$
I(r)=2 \operatorname{Re} I_{+}(r),
$$

где

$$
I_{+}(r)=-\frac{1}{2 \pi i} \int_{c+i 0}^{c+i \infty} d s \frac{\pi e^{\mu \beta s} s^{r}}{\sin (\pi s)} .
$$

При $r>0$ можно положить $c=0$ и тогда

$$
I_{+}(r)=\left(\frac{i}{2 \pi}\right)^{r+1} \int_{0}^{\infty} t^{r} \exp \left[-t\left(\frac{1}{2}+\frac{\mu \beta}{2 \pi i}\right)\right]\left(1-e^{-t}\right)^{-1} d t .
$$

Учитывая, что [22]

$$
\Gamma(s) \zeta(s, v)=\int_{0}^{\infty} t^{s-1} e^{-v t}\left(1-e^{-t}\right)^{-1} d t
$$

получаем

$$
I_{+}(r)=\left(\frac{i}{2 \pi}\right)^{r+1} \Gamma(r+1) \zeta\left(r+1, \frac{1}{2}+\frac{\mu \beta}{2 \pi i}\right)
$$

где $\zeta(s, v)-\zeta$-функция Гурвица.

Равенство (30) является результатом вычисления интеграла (27) для любых значений $r$, кроме $r=-n-1, n=0,1,2, \ldots$. Для $r=-n-1$ интеграл (27) может быть вычислен с помощью вычетов.

С учетом изложенного получаем следуюшее выражение для $\Omega$-потенциала:

$$
\begin{aligned}
\Omega= & \frac{3}{4 \beta}\left\{\frac{4 \pi}{3 \beta_{f}(\varepsilon)}\left(\frac{2 \pi}{E_{0} \beta}\right)^{3 / 2} \operatorname{Re}\left[e^{-3 i \pi / 4} \zeta\left(-\frac{3}{2}, \frac{\mu \beta}{2 \pi i}\right)\right]+\right. \\
& +\omega_{0}+\beta E_{0} \zeta\left(-\frac{4}{3}, \beta_{f}(\varepsilon), \frac{\beta_{f}(\varepsilon)+1}{2}\right)\left(1+e^{-\mu \beta}\right)^{-1}+ \\
& +\sum_{n=2}^{\infty} \frac{(-1)^{n}}{n}\left(\frac{E_{0} \beta}{2 \pi}\right)^{n} \zeta\left(-\frac{4 n}{3}, \beta_{f}(\varepsilon), \frac{\beta_{f}(\varepsilon)+1}{2}\right) \times \\
& \left.\times 2 \operatorname{Re}\left[e^{i \pi n / 2} \zeta\left(n, \frac{1}{2}+\frac{\mu \beta}{2 \pi i}\right)\right]\right\}
\end{aligned}
$$


где

$$
\omega_{0}=\frac{\beta_{f}^{2}(\varepsilon)-2 \beta_{f}(\varepsilon)+3}{24 \beta_{f}(\varepsilon)} \ln \left(1+e^{\mu \beta}\right) \text { при } \quad \mu<0
$$

и

$$
\omega_{0}=\frac{\beta_{f}^{2}(\varepsilon)-2 \beta_{f}(\varepsilon)+3}{24 \beta_{f}(\varepsilon)}\left[\mu \beta+\ln \left(1+e^{-\mu \beta}\right)\right] \text { при } \mu>0 .
$$

В высокотемпературном приближении $(\beta \rightarrow 0)$ главные члены разложения имеют вид

$$
\begin{aligned}
\Omega= & \frac{3 \sqrt{\pi}}{8 \beta_{f}(\varepsilon) E_{0}^{3 / 2} \beta^{5 / 2}}\left(\frac{\sqrt{2}}{4}-1\right) \zeta(5 / 2)+ \\
& +\frac{3 \sqrt{\pi} \mu}{8 \beta_{f}(\varepsilon) E_{0}^{3 / 2} \beta^{3 / 2}}\left(\frac{\sqrt{2}}{2}-1\right) \zeta(3 / 2)+O\left(\beta^{-1}\right),
\end{aligned}
$$

где $\zeta(s)-\zeta$-функция Римана.

Сравнивая этот результат с соответствующим выражением (6), легко заметить существенные различия. Так, $\Omega$-потенциал (32) отличается характером поведения в высокотемпературном приближении $\left(\sim \beta^{-5 / 2}\right)$ от $\Omega$-потенциала (6) для “гармонического осциллятора" ( $\left.\beta^{-3}\right)$. Для последнего первый член разложения (6) соответствует закону Стефана-Больцмана в пространстве размерности $(2+1)$.

Таким образом, в данной работе показано, что в низкотемпературной области $\Omega$-потенциалы для гамильтонианов (1) и (2) имеют схожее поведение. В то жевремя в высокотемпературной области для гамильтониана (2) зависимость $\Omega$-потенциала от температуры имеет сушественное отличие от аналогичной зависимости для гамильтониана (1).

Следует заметить, что для сильно анизотропных кристаллических структур "слоистого" и "цепочечного" типов ранее на основе постулирования закона дисперсии в классической области проводились вычисления для свободной энергии (см., например, [20, $\S 68]$ ). Было показано, что изменение закона дисперсии приводит к сушественному изменению температурной зависимости теплоемкости. В нашем рассмотрении закон дисперсии определяется из нерелятивистской квантовой теории и существенным отличием является истинная двумерность задачи. Последнее обстоятельство важно как для осуществления ВКБ-квантования, так и для нахождения волновых функций системы. Еще одним важным аспектом развитого выше подхода является вычисление волновых функций для гамильтониана (2), в котором переменные не разделяются. Само наличие волновых функций для задачи такого типа может быть интересным при вычислении средних моментов, а также спиновых эффектов и их вкладов в термодинамический потенциал системы. Относительно классической системы, соответствующей гамильтониану (2), следует отметить наличие в ней стохастической компоненты $[10-12,15]$. Возможно, что это влияет на термодинамические свойства системы.

Если вспомнить, что в классической механике высоковозбужденные состояния отвечают, в частности, стохастическому состоянию системы (т.е. сушествует стохастическая компонента решения классических уравнений движения), то возможно, что разница в энергии системы $\Delta E=C \beta^{-3}-C_{1} \beta^{-5 / 2}$ отвечает термодинамической мере вклада этой стохастической компоненты в энергию системы. 


\section{ПРИЛОЖЕНИЕ}

Расчеты $\Omega$-потенциала можно провести с помощью $\zeta$-функции вида

$$
\zeta_{\mathrm{K}}(s, a, b)=\sum_{m, n=0}^{\infty}\left(a\left(m+\frac{1}{2}\right)+b\left(n+\frac{1}{2}\right)\right)^{-s},
$$

которая связана с функцией (21) следующим образом:

$$
\zeta_{\mathrm{K}}(s, a, b)=a^{-s} \zeta\left(s, \frac{b}{a}, \frac{a+b}{2 a}\right)=b^{-s} \zeta\left(s, \frac{a}{b}, \frac{a+b}{2 b}\right)
$$

Приведем некоторые свойства функции $\zeta_{\mathrm{K}}(s, a, b)$, необходимые для вычислений.

Если $a / b$ - иррациональное число, то

$$
\begin{aligned}
\Gamma(s) \zeta_{\mathrm{K}}(s, a, b)= & \frac{2 \pi}{a b \sin (\pi s / 2)}(2 \pi)^{s-2}\left(b^{2-s}+a^{2-s}\right)\left(2^{s-1}-1\right) \zeta(2-s)+ \\
& +\frac{2 \pi}{a b \sin (\pi s / 2)} \sum_{m, n=1}^{\infty}(-1)^{m+n}\left[\left(\frac{2 \pi n}{b}\right)^{s}-\left(\frac{2 \pi m}{a}\right)^{s}\right] \times \\
& \times\left[\left(\frac{2 \pi n}{b}\right)^{2}-\left(\frac{2 \pi m}{a}\right)^{2}\right]^{-1}
\end{aligned}
$$

при $\operatorname{Re} s \in(-2,0)$. Если $a / b$ - рациональное число, то соответствуюшее выражение будет более громоздким, и мы его не приводим. С помощью написанного представления можно убедиться, что функция $\zeta_{\mathrm{K}}(s, a, b)$ имеет простой полюс в точке $s=2$ с вычетом $1 / a b$ и $\zeta_{\mathrm{K}}(0, a, b)=-\left(a^{2}+b^{2}\right) /(24 a b)$.

Кроме того, можно получить следующие функциональные соотношения

$$
\begin{aligned}
\Lambda(s)= & \tilde{\Lambda}(2-s) \\
\Lambda(s)= & 2^{s-1} \Gamma\left(\frac{s+1}{2}\right) \zeta_{\mathrm{K}}(s, a, b) \\
\tilde{\Lambda}(s)= & \frac{1}{\sqrt{\pi}} \Gamma\left(\frac{s}{2}\right) \tilde{\zeta}(s, a, b) \\
\tilde{\zeta}(s, a, b)= & \frac{(2 \pi)^{1-s}}{a b}\left(b^{s}+a^{s}\right)\left(2^{-s}-1\right) \zeta(s)+ \\
& +\frac{2 \pi}{a b} \sum_{m, n=1}^{\infty}(-1)^{m+n}\left[\left(\frac{2 \pi n}{b}\right)^{2-s}-\left(\frac{2 \pi m}{a b}\right)^{2-s}\right] \times \\
& \times\left[\left(\frac{2 \pi n}{b}\right)^{2}-\left(\frac{2 \pi m}{a b}\right)^{2}\right]^{-1} .
\end{aligned}
$$




\section{Список литературы}

[1] Л. Д. Ландау. Собр. трудов. Т.1. М.: Наука, 1969.

[2] W. J. De Haas, P. M. van Alphen. Proc. Netherlands Roy. Acad. Sci. 1930. V. 33. P. 680; P. 1106 .

[3] Д. Шенберг. Магнитные осцилляции в металлах. М.: Мир, 1986.

[4] Я. М. Блантер, М. И. Каганов, Д. В. Посвянский. УФН. 1995. Т. 165. С. 213.

[5] А.С. Виивцев, К.Г. Клименко, Б.В. Магницкий. ЖЭТФ. 1995. Т. 107. С. 307; А. С. Виивцев, К.Г. Клименко. ЖЭТФ. 1996. Т. 109. С. 954.

[6] В. А. Гейлер, В. А. Марәулис, И. В. Чудаев. ЖЭТФ. 1996. Т. 109. № 3. С. 762.

[7] И. М. Лифииц. Избранные труды. Электронная теория металлов. Физика полимеров и биополимеров. М.: Наука, 1994.

[8] Л. Д. Ландау, Е. М. Лифииц. Квантовая механика (нерелятивистская теория). М.: Наука, 1989.

[9] Х. Цикон, Р. Фрезе, В. Кири, Б. Саймон. Операторы Шредингера с приложениями к квантовой механике и глобальной геометрии. М.: Мир, 1990.

[10] Р. Балеску. Равновесная и неравновесная статистическая механика. Т. 1, 2. М.: Мир, 1978.

[11] В. В. Козлов. Симметрия, топология и резонансы в гамильтоновой механике. Ижевск: Изд-во Ижевского гос. ун-та, 1995.

[12] H. Yoshida. Celest. Mech. 1984. V. 32. P. 73.

[13] O. Bohigas, S. Tomsovio, D. Ullmo. Phys. Rep. 1993. V. 223. № 2. P. 43.

[14] M. C. Cutzwiller. Chaos in Classical and Quantum Mechanics. New-York: Springer-Verlag, 1990.

[15] B. Simon. Ann. Phys. 1983. V. 146. P. 209.

[16] В. П. Маслов. Комплексный метод ВКБ в нелинейных уравнениях. М.: Наука, 1977.

[17] В. В. Белов, С. Ю. Доброхотов. ДАН СССР. 1988. Т. 298. № 5. С. 1037.

[18] В. В. Белов, С. Ю. Доброхотов. ТМФ. 1992. Т. 92. № 2. С. 215.

[19] А. С. Виивцев, Н. В. Норин, В. Н. Сорокин. ТМФ. 1996. Т. 109. № 1. С. 107.

[20] Л. Д. Ландау, Е. М. Лифииц. Статистическая физика. Т. 1. М.: Наука, 1995.

[21] Г. Бейтмен, А. Эрдейи. Таблицы интегральных преобразований. Т. 1. М.: Наука, 1969.

[22] Г. Бейтмен, А. Эрдейи. Высшие трансцендентные функции. Т. 1. М.: Наука, 1973.

Поступила в редакцию 4.II.1998 г. 